\title{
Phenotypic classification of multidrug-resistant Klebsiella through MALDI-TOF mass spectrometry
}

\author{
Anna Di Taranto, ${ }^{1}$ Rosella De Nittis, ${ }^{1}$ Marco Fagioni, ${ }^{2}$ Tiziana Rollo, ${ }^{1}$ Paola Cella, ${ }^{1}$ \\ Raffaele Antonetti ${ }^{1}$ \\ ${ }^{1}$ Department of Clinical Pathology, Analysis Laboratory II, Ospedali Riuniti of Foggia University \\ Hospital; ${ }^{2}$ Bruker Daltonics s.r.l., Macerata, Italy
}

\section{Summary}

Background. Resistance to carbapenems in Klebsiella pneumoniae infections could compromise therapy efficiency in hospitals. In Italy, the percentage of $K$. pmeumoniae resistant to carbapenems blood-isolated is $35 \%$.

Materials and Methods. Seventy-three multidrug-resistant $K$. pneumoniae clinical isolates coming from Foggia (Italy) have been phenotypically classified through matrix assisted laser desorption/ionization-time of flight mass spectrometry between February and May 2014 in order to locate an eventual infectious outbreak.

Conclusions. Classification of microbial isolates is essential to this purpose and starts from phenotypic methodologies to the most complex genotypic methods. Among these, mass spectrometry utilization appears to be a simple, useful and quick instrument in a clinical microbiology laboratory.

\section{Introduction}

Resistance to carbapenems, growing in severe Enterobacteria infections and especially in Klebsiella pneumoniae infections, com-

Correspondence: Anna Di Taranto, Dipartimento di Patologia Clinica, Laboratorio Analisi, Azienda Mista Ospedaliero-Universitaria 00.RR Foggia, Viale Luigi Pinto 1, Foggia, Italy.

Tel.: +39.800 .102 .171 .

E-mail: aditaranto@ospedaliriunitifoggia.it

Key words: Klebsiella pneumonia; carbapenems; matrix assisted laser desorption/ionization-time of flight mass spectrometry.

Contributions: the authors contributed equally.

Conflict of interest: the authors declare no potential conflict of interest.

Received for publication: 21 January 2015.

Accepted for publication: 29 May 2015.

(c) Copyright A. Di Taranto et al., 2015

Licensee PAGEPress, Italy

Microbiologia Medica 2015; 30:5015

doi:10.4081/mm.2015.5015

This article is distributed under the terms of the Creative Commons Attribution Noncommercial License (by-nc 3.0) which permits any noncommercial use, distribution, and reproduction in any medium, provided the original author(s) and source are credited. promises the therapy efficiency in the hospital environment and leads to a major degree of mortality if compared to sensitive bacteria (5). In Italy, which is the most affected State in Europe after Greece and Cyprus, the percentage of $K$. pmeumoniae resistent to carbapenems blood-isolated was 35\% in 2013 according to European Antimicrobial Resistance Surveillance Network (2). After locating patients who are affected or colonized by these pathogenic agents, it is essential to execute efficient inspections (1) and to set up epidemiological investigations to determine infections sources and their diffusion (4), in order to contrast the hospital carbapenems resistance increase (3). For this purpose, the phenotypic classification of clinical isolates of multidrug-resistant $K$. pneumoniae through matrix assisted laser desorption/ionization-time of flight mass spectrometry (MALDI-TOF MS), a simple and low-cost methodology compared to genotypic investigation, plays a key role.

\section{Materials and Methods}

All the multidrug-resistant (MDR) $K$. pneumoniae clinical isolates coming from wards of Azienda Ospedaliera Universitaria 00.RR of Foggia (Italy) have been phenotypically classified through MALDI-TOF MS in the period between February and May 2014, in order to locate an eventual infectious outbreak and consequently strengthen the adoption of appropriate countermeasures of infection control.

Seventy-three clinical isolates of $K$. pneumoniae have been analyzed in the period between February and May 2014, and using the BD Phoenix ${ }^{\mathrm{TM}}$ (Becton Dickinson, BD Diagnostic Systems, Sparks, MD, USA) identification system and antibiogram, they showed resistance to several carbapenems. Patients were 38 male (52\%) and 35 female with an average age of 70 years; they came from Intensive Care Unit (36\%), Internal Medicine (23\%), Physiatry (19\%), Nephrology (18\%), Surgery (2\%), Hematology (2\%) Departments; clinical items were urines $(66 \%)$, blood $(19 \%)$, washing bronchial (12\%) and pus (3\%). The 14 blood samples, coming from Intensive Care unit in the $85 \%$ of cases, have been further analyzed with GeneXpert $^{\mathrm{TM}}$ Carba-R assay (Cepheid, Sunnyvale, CA, USA), that found in all of them the KPC sequence. The isolates, after defrosting and passage on Agar blood Columbia (Becton Dickinson) and extracted with standard procedure, have been analyzed with MALDITOF MS Microflex ${ }^{\mathrm{TM}}$ LT (Bruker Daltonics, Bremen, Germany), reiterating 16 times for each sample to exclude casual effects. Spectra have been analyzed with ClinProt Tools 3.0 (Bruker Daltonics) software with the purpose of building a resuming dendrogram and for a statistical analysis of clusters based on principal component analysis (PCA) variance. 


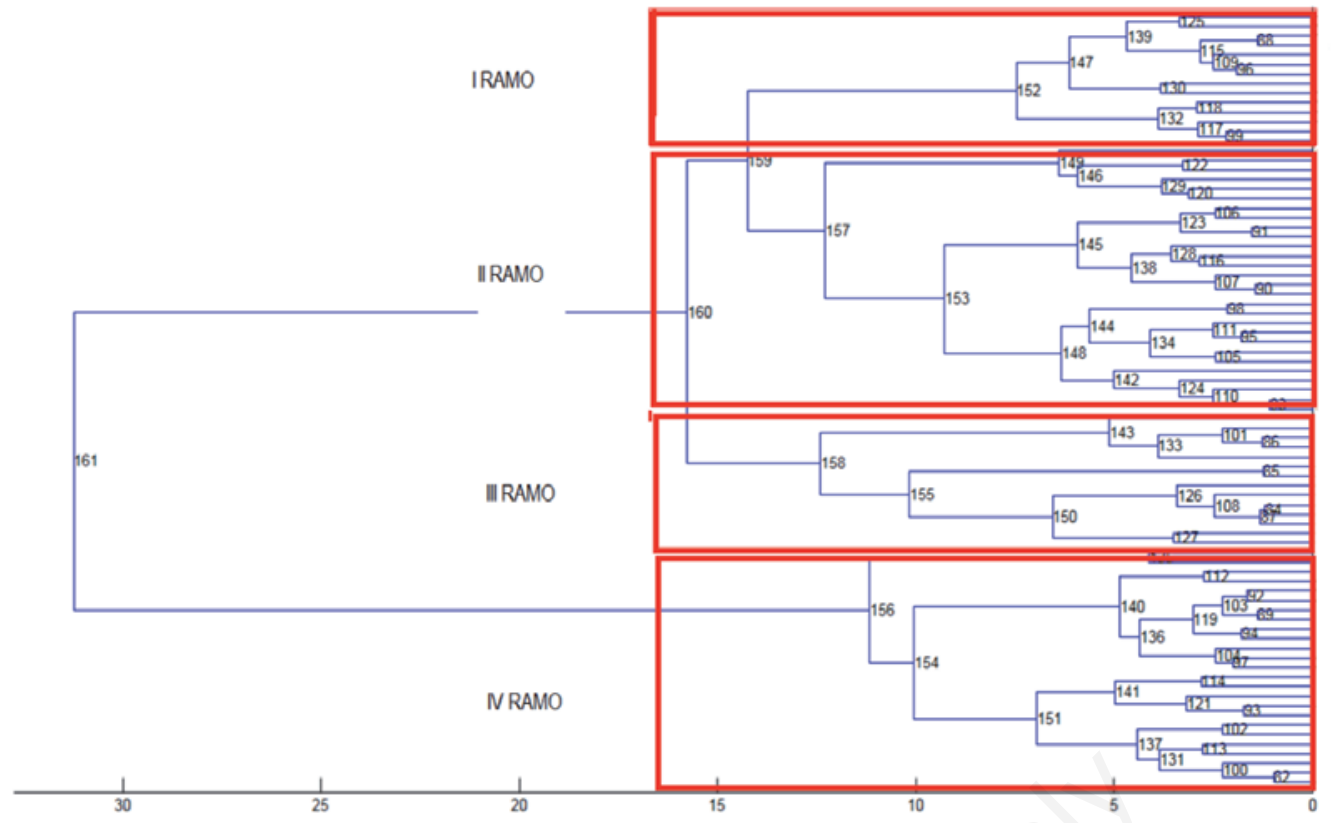

Figure 1. The dendrogram shows four distinct groups presenting a high similarity.

\section{Results}

The dendrogram (Figure 1) shows four distinct groups presenting a high similarity, denoted with numbers from I to IV; in Physiatry and Nephrology, where all samples were urines, there is not a unique group prevalence. In the medical wards, where samples were $65 \%$ urines, stem II prevails in February and stem III in April and May. Even in Intensive Care units, where samples were $39 \%$ coming from blood, $34 \%$ LAB, $19 \%$ urines and $8 \%$ exuded, from February to April group II prevails (35\%), while in May group III does (27\%). This trend is confirmed in every kind of sample, particularly for blood. Statistical analysis of PCA (Figure 2) highlighted the presence of two distinct clusters (green

\section{CR1 PCA}

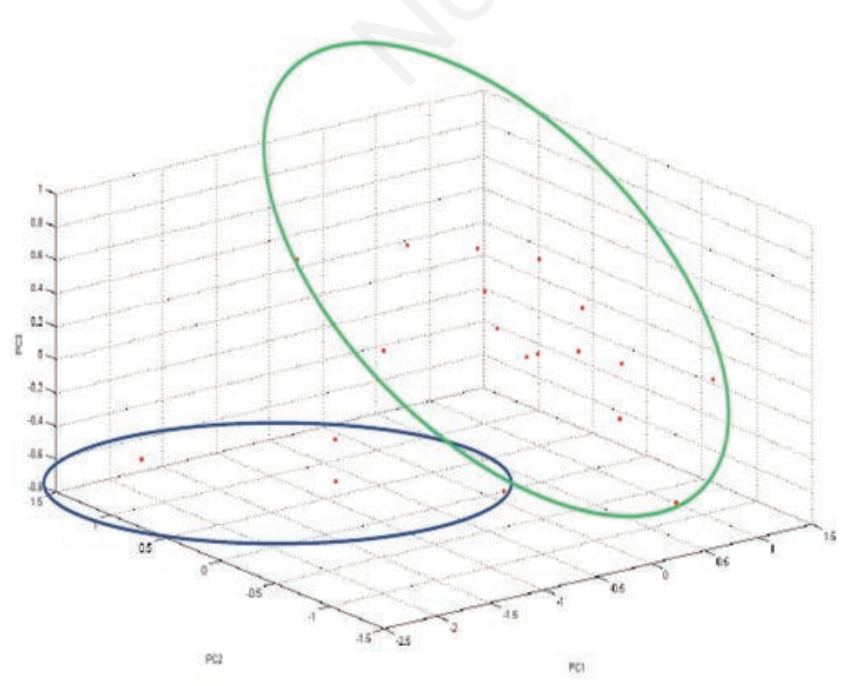

Figure 2. Statistical analysis of PCA highlights the presence of two distinct clusters (green and blue ones) in Intensive Care 1. and blue ones) in Intensive Care 1, from which the major number of logs were coming, and consequently a possible infectious outbreak.

\section{Discussion and Conclusions}

Classification of microbial isolates, essential to demonstrate a possible infectious outbreak, starts from phenotypic methodologies to reach the most complex genotypic methods. Among phenotypic methods, recently, mass spectrometry utilization, analyzing proteic spectra of isolates, appears to be a simple, useful and quick instrument for MDR stems diffusion prevention in a clinical microbiology laboratory. Such technique allowed to evaluate if in a given ward, $K$. pneumoniae MDR isolates did or did not belong to a unique circulating stem and consequently to foresee the need to strengthen prevention protocols of infections due to clinical support operations.

\section{References}

1. Cuzon G, Naas T, Nordmann P. KPC carbapenemases: what is at stake in clinical microbiology? Pathol Biol (Paris) 2010; 58: 39-45.

2. European Centre for Disease Prevention and Control. European Antimicrobial Resistance Surveillance Network EARS-Net. Available from: http://ecdc.europa.eu/en/activities/surveillance/ EARS-Net/Pages/index.aspx. Accessed on: January 2015.

3. Berrazeg M1, Diene SM, Drissi M, et al. Biotyping of multidrugresistant Klebsiella pneumoniae clinical isolates from France and Algeria using MALDI-TOF MS. PLoS One 2013; 8: e61428.

4. Murray PR. Matrix-assisted laser desorption ionization time-offlight mass spectrometry: usefulness for taxonomy and epidemiology. Clin Microbiol Infect 2010; 16; 1626-30.

5. Patel G, Huprikar S, Factor SH, et al. Outcomes of carbapenemresistant Klebsiella pneumoniae infection and the impact of antimicrobial and adjunctive therapies. Infect Control Hosp Epidemiol 2008; 12: 1099-106. 